\title{
Criminologie
}

\section{Le crime économique}

\section{Jacques Marquis}

Volume 10, numéro 1, 1977

La criminalité des affaires au Québec

URI : https://id.erudit.org/iderudit/017067ar

DOI : https://doi.org/10.7202/017067ar

Aller au sommaire du numéro

Éditeur(s)

Les Presses de l'Université de Montréal

ISSN

0316-0041 (imprimé)

1492-1367 (numérique)

Découvrir la revue

Citer cet article

Marquis, J. (1977). Le crime économique. Criminologie, 10(1), 79-93.

https://doi.org/10.7202/017067ar d'utilisation que vous pouvez consulter en ligne.

https://apropos.erudit.org/fr/usagers/politique-dutilisation/ 
A. À la Sûreté du Québec, on définit le crime économique comme suit :

Un crime commis dans l'une ou l'autre des étapes du processus économique, soit dans la production, la distribution, le commerce de biens et services, à l'exclusion :

a) crimes contre la personne ;

b) crimes contre la propriété.

i) Le vol qualifié, le meurtre, l'extorsion, les délits sexuels et tous les autres crimes qui touchent à la personne relèvent de l'escouade des crimes contre la personne.

ii) L'incendie criminel, le vol simple et par effraction, le vol d'autos et tous les autres crimes contre la propriété relèvent de l'escouade des crimes contre la propriété.

B. Les types de crimes économiques

Les types de crimes suivants sont donc identifiés comme relevant du crime économique :

1. La faillite. En particulier l'aliénation de biens avec l'intention de frauder des créanciers, art. 350 du Code criminel.

2. Les ventes pyramidales. Système ou plan en vertu duquel le participant peut recevoir des avantages du fait qu'il recrute d'autres participants, art. 189-1-e du Code criminel.

3. Les valeurs mobilières. Influencer illégalement les actions boursières, l'agiotage sur les actions, cacher frauduleusement des titres et enregistrer frauduleusement des titres, art. 338, 340, 341, $342,343,344,355$ et 358 du Code criminel.

4. Les valeurs immobilières. La vente illégale d'un immeuble et la vente sous de fausses représentations de terrains et immeubles, art. 338 et 345 du Code criminel.

5. La contrefaçon de monnaie. Toute la contrefaçon de monnaie prévue à la partie $\mathrm{X}$ du Code criminel, art. 406 à 420 du Code criminel. 
6. La corruption. Nous entendons toutes les formes de corruption au niveau politique, fonctionnaire et entreprise privée, telles que prévues aux art. 383, 108, 109, 111, 112, 113, $114 \mathrm{du}$ Code criminel.

7. La malversation. Tout détournement de fonds ou de biens dans l'exécution d'une fonction ou d'une charge.

8. La fraude par carte de crédit. L'utilisation de fausses cartes de crédit ou de cartes de crédit volées. Plus souvent qu'autrement, ceci se fait par des groupes organisés, art. 301.1 du Code criminel.

9. La fraude par chèque. L'utilisation de chèques faux, volés ou contrefaits. Des groupes se spécialisent dans I'utilisation de tout le système de traite bancaire pour frauder sur une grande échelle, art. 320 du Code criminel.

10. L'escroquerie. Toute action qui a pour résultat l'obtention de quelque chose par moyens frauduleux. Le modus operandi pouvant varier selon l'imagination, art. 320 et 324 du Code criminel.

Le degré de complexité de ces crimes peut varier du relativement simple au crime complexe très sophistiqué. La majorité des crimes enquêtés par notre unité tombe dans la catégorie des crimes complexes.

C. Les victimes

Il y a trois (3) catégories de victimes :

1. Les citoyens, qui peuvent avoir été victimes individuellement ou en groupe.

2. Les entreprises commerciales et financières.

3. Les gouvernements qui sont sûrement les plus grandes victimes des criminels à collet blanc.

Il est à noter que ces victimes ne sont pas toujours conscientes de leur statut de victime. Par exemple, dernièrement, lors du démantèlement du réseau de fraudeurs dans la livraison d'huile à chauffage, les clients des trois (3) catégories précitées étaient des victimes sans le savoir.

D. Les criminels à collet blanc

Il y a selon nous, deux (2) catégories de criminels à collet blanc, celui qui agit seul et ceux qui vont conspirer la commission du crime. 
La majorité des cas sur lesquels on fait enquête se situe dans la deuxième catégorie. La majorité des crimes à collet blanc implique d'ailleurs une relation interpersonnelle. L'élément essentiel de ces crimes étant la tromperie, le mensonge, l'abus de confiance.

Dans l'ensemble, ces criminels font preuve d'une capacité de communications interpersonnelles plus importante que celle qu'on constate chez d'autres types de criminels ${ }^{1}$.

Compte tenu de notre expérience personnelle en ce qui a trait aux injures qu'on reçoit des criminels reconnus comme violents, nous avons été très surpris lors de notre première enquête majeure sur un « fraudeur» d'être reçu par celui-ci avec un sourire et une tasse de café.

En général, les relations policiers-suspects dans les enquêtes concernant les crimes économiques sont presque cordiales et tout du moins très professionnelles. Elles semblent s'inscrire dans le cadre d'une sorte de respect mutuel.

\section{LA POLICE (les escouades spécialisées)}

A. Suite à l'identification par Sutherland ${ }^{2}$ du crime à collet blanc comme type bien spécifique de criminalité, la police a été lente à réagir, tout comme les criminologues d'ailleurs. Ce n'est qu'au début des années 60 que les corps policiers canadiens se sont dotés d'unités d'enquêtes spécialisées sur la fraude et le crime économique en général.

Le 18 août 1975, la Sûreté du Québec, après étude, a décidé de se doter de deux escouades de crimes économiques : une à Montréal, qui a débuté avec 27 policiers et une à Québec, qui comprenait 13 policiers. Le choix du personnel a été fait à la suite de la demande de volontaires parmi les policiers de la Sûreté du Québec, avec indication des qualifications de base.

Le policier désirant travailler dans le domaine du crime économique devait avoir huit ans d'expérience dont deux ans sur les enquêtes, une scolarité du niveau secondaire, au minimum, être bilingue et disposer d'une connaissance pratique ou théorique de l'économique, de la comptabilité, du système bancaire, des affaires, etc. Une formation académique valable pouvait permettre

1. Voir bibliographie.

2. Voir bibliographie. 
à un policier ayant six ans uniquement d'expérience de solliciter le poste.

Par la suite, plus de cent candidats ont été interviewés dont quarante furent sélectionnés. Ce groupe constitue les effectifs actuels d'enquêteurs sur les crimes économiques à la Sûreté du Québec.

La gendarmerie royale du Canada possède depuis 1967 une section des délits commerciaux avec des unités dans chaque province canadienne. La section de Montréal des délits commerciaux de la G.R.C. compte plus de cent policiers et l'ensemble des sections à travers le Canada compte près de quatre cents policiers. Ces sections sont coordonnées par un bureau central à Ottawa et comprennent des unités bien organisées disposant d'un personnel compétent.

Le Service de la police de la Communauté urbaine de Montréal (S.P.C.U.M.) dispose d'une unité d'enquêtes des activités frauduleuses, qui compte plus de cinquante (50) policiers. Cette unité s'acquitte d'un fort volume de travail ayant à répondre à des plaintes d'un bassin de population frôlant le deux millions de citoyens et du centre des affaires pour la province.

\section{B. Manque d'effectifs}

Il va sans dire que compte tenu de la mission de la police en ce qui a trait aux crimes économiques, soit la prévention, la détection et l'appréhension (Loi de police, art. 16), les quelque cinquante membres de la S.Q. assignés à ces fonctions ne suffisent pas à la tâche.

La police est bien consciente des critiques qu'on lui adresse à ce sujet et que Jean-Claude Leclerc, entre autres a résumé dans son article «L'administration dépolitisée de la justice », publié dans le journal Le Devoir ${ }^{3}$.

«À cet égard, l'autonomie de la police et l'indépendance de la magistrature peuvent demeurer longtemps des principes aussi respectables que stériles si le gros des effectifs policiers, par exemple, est affecté à la surveillance de routes, des universitaires ou des grévistes, plutôt qu'à l'examen des faillites frauduleuses, des évasions fiscales, des corruptions municipales et scolaires, $j$ 'en passe et des meilleures $\gg$.

3. Voir bibliographie. 
À la Sûreté du Québec, on prévoit une augmentation considérable des effectifs d'enquêteurs sur les crimes économiques, mais ceci se fera d'une façon ordonnée et systématique sur une période d'un certain nombre d'années. Il faut souligner que l'étendue du problème n'est pas encore évaluée. Nous croyons que les crimes identifiés ne représentent que le sommet du glacier.

Le personnel sera donc encore accru selon la capacité de ces unités d'absorber du sang nouveau, d'entrainer et de former des officiers à des techniques d'enquêtes très différentes de l'enquête policière traditionnelle.

\section{La formation pluridisciplinaire}

L'enquêteur sur les crimes économiques doit posséder de bonnes connaissances des techniques traditionnelles d'enquêtes, plus une capacité d'adaptation à des milieux fort différents du criminel habituel.

L'Institut de police du Québec a formé un comité de travail pour développer un cours spécialisé en matière d'enquêtes sur les crimes économiques. Ce cours qui a commencé en automne 1976 contiendra les sujets énumérés au tableau I, p. 84.

Le cours est donné à l'Institut de police avec des personnes ressources spécialisées dans chacune de ces matières. Il s'adresse aux enquêteurs possédant déjà l'expérience d'enquête, ayant suivi les cours d'enquêtes judiciaires et ayant le cours de base en comptabilité d'une université du Québec. Sa durée est d'un mois à plein temps.

\section{LA TECHNIQUE D'ENQUETE SUR LES CRIMES ECO- NOMIQUES}

Peu importe l'enquête, les principes de base sont toujours les mêmes, il y a un crime, une victime, des pièces à conviction et des témoignages. Dans le cas des crimes économiques, lorsqu'un citoyen porte plainte, il n'est pas toujours évident qu'il y a crime. Car, l'acte criminel est généralement enseveli dans des transactions commerciales normales. Un des premiers objectifs sera d'établir s'il y a effectivement eu crime. Très souvent une enquête débutera sans victime ni plaignant. Dans un cas de corruption, par exemple, qui est la victime? 
Conséquemment, l'enquêteur de crimes économiques devra travailler avec beaucoup de méthode pour éviter de poursuivre une enquête inutilement. Nos recherches nous ont permis d'établir quatre phases à une enquête sur les crimes économiques. (Voir annexe I).

\section{La phase renseignement ( $I^{\text {ère }}$ phase)}

Dans toute enquête, il doit toujours y avoir une phase de renseignement qui précède toutes les autres. La phase renseignement peut durer une heure (dans le cas d'un plaignant possédant les preuves à l'appui de ses affirmations) ou plusieurs mois, si tous les moyens à notre disposition sont utilisés.

Dans cette phase de renseignement, on fait appel au plaignant, à l'indicateur, à l'interception des communications privées (art. 178.1 du Code criminel) et aux organismes policiers et civils susceptibles de nous renseigner sur les suspects ou compagnies, toujours selon les besoins de la cause.

\section{TABLEAU 1}

Liste des matières

Affaires municipales

Cas de fraudes

Communications privées, 178.1 C.C.C.

Les compagnies

La comptabilité pour la Cour

La corruption de fonctionnaires

Le montage de dossiers

Le droit civil

Le droit pénal spécialisé

Les faillites

Les faux chèques et cartes de crédit

La filature

Le laboratoire de police scientifique

Les perquisitions

La protection du consommateur

La psychologie du criminel à collet blanc

Les sources d'information

La structure financière du gouvernement

Le système bancaire canadien

Les valeurs mobilières

Les valeurs immobilières

Les ventes pyramidales illégales

Évaluation

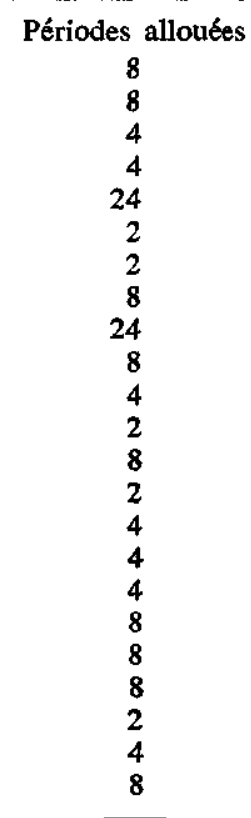

Total : 160 
Dans les dossiers les plus complexes, il sera nécessaire de monter un système de dossiers temporaires. Ces dossiers serviront lors de la phase de perquisitions et d'enquêtes. Ils pourront être épurés et détruits par la suite.

Avant de passer à la phase de perquisition, il faut établir s'il y a eu crime. Si oui, il faut identifier l'article du Code criminel qui s'applique.

Finalement, il faut faire la preuve. L'enquêteur se doit de connaître les exigences du Code criminel. Au besoin, il doit consulter un procureur. Oublier qu'à ce stage-ci un élément essentiel à la preuve peut avoir des conséquences sérieuses à la fin de l'enquête.

\section{La phase perquisition ( $2^{\circ}$ phase $)$}

La phase perquisition nous permet de savoir qui possède et à quel endroit se trouvent les pièces à conviction dont nous aurons besoin pour faire la preuve du crime que nous avons identifié.

Il y a eu une procédure judiciaire, le bref de certiorari, qui est utilisé pour faire casser un mandat de perquisition pour vice de forme ou vice de fonds. Dans le cas des crimes économiques, nous avons déjà eu des mandats de perquisition qui furent cassés.

Sommairement, le mandat de perquisition doit contenir :

1) la description de l'acte criminel ;

2) le modus operandi (avec noms et dates) ;

3) les objets recherchés ;

4) les motifs de l'enquêteur (suffisamment de détails);

Tout mandat de perquisition doit être obtenu par l'enquêteur lui-même. Dans tous les cas, il faut consulter un procureur de la Couronne.

Lorsqu'il y a plusieurs perquisitions simultanées, il faut préparer un plan qui contiendra :

a) un état de situation;

b) les objectifs des perquisitions ;

c) la liste des endroits qui seront perquisitionnés ;

d) le plan tactique des perquisitions (horaire et ressources physiques) ; 
e) la provenance des effectifs ;

f) l'assignation des effectifs ;

g) la codification des pièces à conviction.

Finalement, il ne faut pas oublier de demander au juge la garde légale des pièces à conviction qui doit être renouvelée tous les 90 jours. Les renseignements et les pièces à conviction en main, nous pouvons passer à la phase de l'enquête.

\section{La phase investigation ( $3^{*}$ phase)}

Après étude des renseignements et des pièces à conviction, il faut préparer le plan d'enquête. Dans une enquête de crime économique, le danger est de s'étendre au point de perdre de vue l'objectif premier. Le plan d'enquête est essentiel à sa bonne conduite. C'est aussi un gage de succès.

Tous les trucs de l'enquête traditionnelle seront utilisés, l'interrogatoire, la confrontation, les laboratoires de police scientifique, etc. À ceci, on peut ajouter l'assistance de professionnels tels les comptables, les ingénieurs, les juristes, etc. Un mot sur l'utilisation d'experts professionnels. Ces derniers doivent obtenir de l'enquêteur un mandat précis, un objectif à atteindre et tous les matériaux dont ils ont besoin. Ces experts doivent être consultés au maximum, mais ils ne doivent pas remplacer l'enquêteur qui est le seul responsable de la conduite de son enquête. C'est l'enquêteur qui finalement aura à répondre de son enquête.

Dans tous les dossiers complexes, le procureur de la Couronne doit être informé de l'évolution de l'enquête, de sorte que lorsque l'enquêteur soumettra son rapport, il le soumettra à un procureur réceptif et déjà sensibilisé sur la nature du crime et ses implications juridiques.

Pour les enquêtes qui se prolongent pendant plusieurs mois, il est essentiel que l'enquêteur soumette un rapport progressif mensuel. Ce rapport contiendra une description des cas d'enquêtes, leur progrès et le plan d'action pour le mois à venir.

Finalement, avant de rédiger son rapport, l'enquêteur consciencieux se soumettra à une séance contradictoire, c'est-à-dire qu'il dévoilera sa preuve à ses confrères de travail qui se feront les avocats du diable. Toute faiblesse dans la preuve devrait alors ressortir et l'enquêteur pourra compléter son enquête avant de passer à la phase étalement. 


\section{La phase étalement (46 phase)}

Cette phase consiste à étaler la preuve devant les autorités de la police, les procureurs de la couronne et les tribunaux. L'enquêteur, en préparant son rapport, doit en venir à une conclusion et la définir à la fin de son rapport.

Ces rapports d'enquête sont plus ou moins longs, selon le degré de complexité de la preuve, mais le rapport doit être construit de façon à pouvoir être facilement lu. Tout rapport dépassant une douzaine de pages doit être précédé d'un index.

Le rapport est divisé de la façon suivante :

1) index;

2) sommaire ;

3) déclarations ;

4) preuve technique ;

5) annexes.

Le sommaire doit permettre au lecteur de visualiser en peu de temps l'ensemble du crime et ses auteurs. Les déclarations peuvent être dactylographiées au long ou résumées avec référence à la déclaration en annexe. Les annexes doivent être classées logiquement et numérotées pour référence facile.

Certains rapports sont construits de façon qu'il n'est pas nécessaire de faire un précis de la preuve. Par contre, il se peut qu'un procureur exige le précis de la preuve qui sera alors traditionnel.

C'est le rôle de l'enquêteur de présenter le produit de son enquête au procureur de la Couronne. Ce sera aussi son rôle d'aider le procureur à présenter la preuve devant le tribunal.

Finalement, l'enquêteur est responsable de la disposition des pièces à conviction avant la fermeture du dossier.

\section{LES DIFFICULTÉS DE L'ENQUÊTE EN MATIÈRE DE CRIMES ECONOMIQUES}

A. Les silences du milieu

Les hommes d'affaires aiment bien la police, mais pour protéger leurs biens contre les voleurs. Quand on se présente chez certains hommes d'affaires pour enquêter sur des transactions commerciales suspectes, ils deviennent muets. Ils réclament la présence d'un avocat. Un confrère me disait dernièrement en 
discutant d'un dossier difficile : « Tu lèves une couverte et il y a un avocat là-dessous $\gg$.

Certes, il y a beaucoup d'hommes d'affaires qui collaborent avec la police, sans hésitation, mais disons qu'en général, il faut presque toujours se munir de mandats de perquisition pour consulter des documents que la plupart des citoyens nous exhibent sans hésitation.

Les banques s'appuient sur l'article de la loi des banques pour nous rendre la vie souvent difficile. Beaucoup de gérants de succursales bancaires sont complices par complaisance, complicité non criminelle, mais tout au moins morale. Certains se laissent aller au désir d'augmenter leur chiffre d'affaires au point de servir d'outil involontaire aux fraudeurs.

Les services de l'impôt se réfugient aussi derrière leurs lois pour garder leurs distances avec les escouades de crimes économiques. Ceci bien sûr à l'avantage de criminels à collet blanc qui sont millionnaires et ne paient pratiquement pas d'impôt. Depuis quelques années, une section de lutte contre le crime organisé a été ouverte à la section des enquêtes spéciales.

Mais qu'est-ce que le crime organisé ?

Comme on a pu le voir, la majorité des crimes économiques sont des crimes organisés. La logique de l'article sur le secret des rapports d'impôt implique que les citoyens doivent déclarer tous leurs revenus, y compris ceux qui sont de source criminelle. C'est à se demander, si un criminel devait rapporter des revenus provenant du fruit d'un crime, s'il n'y aurait pas une composition avec un acte criminel (129 C.C.C.) ? Le service de l'impôt au service du public ne devrait-il pas rapporter à la police les crimes qu'il détecte?

B. La frontière entre le droit civil et le droit criminel

Dans notre travail quotidien, nous étudions des transactions commerciales qui sont à la frontière du droit civil et du droit criminel. Certaines transactions sans être criminelles sont hautement immorales. Ceci bien sûr fera toujours partie de notre paysage et nous ne pouvons qu'espérer une évolution du droit vers de plus en plus de précisions, car nous avons abandonné l'idée d'une amélioration de la morale. 
C. Les décisions des cours criminelles

Quel policier ne se plaint-il pas des sentences?

Ceci est dû au fait que les policiers sont plus près des victimes et de l'acte criminel que tout autre agent de la justice. Mais, dans les crimes économiques, les sentences sont vraiment faibles.

Le président d'un syndicat a été accusé par nous d'avoir fraudé son local de $\$ 2400$, en forgeant lors d'une grève l'an dernier, les noms de faux piqueteurs. Il se reconnaît coupable. Pour cet abus de confiance et cette fraude dans des moments de grandes difficultés pour le syndicat, le juge le condamne à trois ans, mais c'est une sentence suspendue et lui accorde le privilège de ne pas avoir de dossier criminel en vertu de l'article $662.1 \mathrm{du}$ Code criminel. L'accusé peut même continuer à bénéficier des fruits du crime et exercer son métier jusqu'à la prochaine grève, et alors? La Couronne a décidé de ne pas appeler de cette décision et cela à notre grand désarroi. Tout le travail que nous avons dû faire en milieu syndical (qui n'est pas des plus réceptifs à la police) a été en fait immobile. Que dire de notre plaignant qui n'appellera sûrement jamais plus la police...

Ce président de syndicat n'avait-il pas une connaissance de l'acte criminel beaucoup plus grande que plusieurs jeunes qui commettent des délits sans trop savoir ce qu'ils font? Et pourtant comparez les sentences!

Ceci n'est qu'un exemple. Nous pourrions en écrire des pages à ce sujet. Je crois à la bonne foi des juges, mais à une mauvaise connaissance de leur part du criminel à collet blanc. C'est en toute connaissance de cause et de sang-froid que le collet blanc commet son crime, bien plus que tout criminel répondant à des instincts de violence.

\section{Les professionnels peuvent être des dupes}

Un fraudeur se présente chez un comptable avec des chiffres de son invention et demande au comptable de lui préparer un bilan et un état de revenus et de dépenses. Le comptable s'exécute et, conformément aux instructions de son ordre des comptables, indique qu'il n'a pas fait la vérification physique. Cette mise en garde dans les rapports financiers est insuffisante. Plusieurs seront victimes de ces travaux qui ont l'apparence de documents dûment 
vérifiés par un professionnel. Les comptables devraient s'abstenir de compléter ces rapports et d'y apposer leurs noms s'ils n'ont pas la certitude morale qu'ils contiennent toute la vérité. Il serait préférable qu'une inspection physique des actifs ait été faite, de même que du passif.

Il en va de même pour les notaires, avocats, ingénieurs, etc. Des professionnels ont une responsabilité morale du produit de leur expertise. Ils doivent être conscients des dommages qui pourraient être causés et prendre les mesures qui s'imposent pour éviter que leurs travaux ne conduisent à faire des victimes.

\section{E. Des crimes traités comme infractions}

Des médecins soumettent des réclamations totalement injustifiées à l'assurance-maladie. $Y$ aurait-il eu fraude et fabrication de faux? Des procédures sont prises en vertu d'une loi provinciale, mais, pour les autres citoyens, de tels gestes seraient des actes criminels et ceux-ci auraient un dossier criminel.

Des centaines de causes pour fraude fiscale sont portées devant les tribunaux chaque année. $Y$ aurait-il eu fraude et fabrication de faux, soit des actes criminels?

Pour les mêmes actes dans des domaines autres que celui de l'impôt, un citoyen serait accusé devant un tribunal criminel et aurait un dossier. Pourtant, les causes de fraude fiscale ne touchent pas les couches inférieures de la société.

Un fonctionnaire ou un employé de l'industrie soumet de fausses factures dans ses comptes de dépenses ; on exige un remboursement et on lui inflige une suspension, mais pour les mêmes gestes, des citoyens sont accusés devant un tribunal criminel et font de la prison.

Pourquoi la justice n'est pas la même pour tous?

C'est pourquoi nous disions au début que nous ne connaissions que la pointe de l'iceberg. Rappelons que la fraude c'est du vol. C'est un vol par fausses représentations. Si la victime avait su la vérité, elle ne se serait jamais départie de son argent ou de son bien. Il y a donc beaucoup de travail de sensibilisation à faire dans ce domaine. 


\section{F. Les contrats " cost-plus »}

Pour terminer, j'aimerais traiter d'une évolution dans le monde des affaires qui représente un terrain fertile pour le criminel à collet blanc. Avec l'arrivée de l'inflation, dans les deux taux les entreprises exigent des contrats $<$ cost-plus $\gg$. Ces contrats déterminent les coûts de travaux aux prix de revient, plus un pourcentage pour les frais de gérance.

Il y a peu de temps, tous les contrats se négociaient à un prix fixe. Le contracteur prévoyait ses coûts d'achats de matériaux, de main-d'œuvre, des frais de gérance et se gardait toujours une marge de manœuvre pour les imprévus et l'inflation. Il se devait d'être prudent, car si le contrat lui coûtait trop cher, il perdait de largent, ce qui pouvait même le conduire à la faillite. Il se devait donc de surveiller ses coûts. C'est la base de la libre entreprise.

Le contrat * cost-plus, vise à combattre l'inflation. Une formule est énoncée dans le contrat pour déterminer le coût de revient, généralement, les coûts d'achats de matériaux, de maind'œuvre et du transport. Ces coûts sont payables à la facturation à l'entrepreneur, plus un pourcentage pour ses frais de gérance et son « know-how». Cette formule est de la dynamite pour ne pas dire de l'encouragement à profiter de la situation.

À titre d'exemple, disons que quelqu'un décide de construire une maison de rapport de huit logements, à un coût approximatif de $\$ 100000$. Il signe un contrat \& cost-plus * avec un contracteur en construction. Le prix sera déterminé comme suit :

a) le total des factures des matériaux utilisés pour construire la propriété ;

b) le total des salaires payés aux ouvriers ;

c) $10 \%$ en frais de gérance.

\section{Les désavantages}

1) Il n'y a aucun encouragement à limiter les coutts.

2) C'est le contracteur qui achète les matériaux. Par hasard, il est aussi propriétaire d'une entreprise qui vend des matériaux de construction et qui porte un nom totalement différent. Il achète donc ces matériaux de cette entreprise au prix le plus haut possible. 
3) Plus il paiera cher les matériaux, plus son entreprise secondaire fera de profits.

4) Plus il paiera cher les matériaux, plus il fera de l'argent en frais de gérance.

5) Plus les ouvriers travailleront lentement et plus il fera de l'argent en frais de gérance.

La propriété coûtera $\$ 150000$ au lieu de $\$ 100000$ prévu et le concerné blâmera l'inflation et les travailleurs.

Cet exemple est simpliste, mais tel est le principe. Peu importe la complexité des clauses du contrat « cost-plus », l'encouragement à contrôler les coûts diminue et celui qui accorde le contrat devrait engager des experts en contrôle des coûts, mais, il sera quand même victimisé.

La structure de l'économie dans laquelle nous vivons implique un profit pour le travail et le risque assuré par l'entreprise privée. Le contrat « cost-plus » est un risque réel pour celui qui l'accorde. Il risque, en effet, de payer très cher.

\section{BIBLIOGRAPHIE}

GOUDREAU-TOUTANT, Cécile, Etude comparative de la capacité interpersonnelle chez deux groupes de fraudeurs, thèse de maîtrise en criminologie. Montréal, Université de Montréal.

SUTHERLAND, Edwin H. (1949), White Collar Crime, New York, HoltRinehart-Winston.

LECLERC, Jean-Claude (1976), - L'administration dépolitisée de la justice », éditorial in le Devoir. 
ENQUETTES DE CRIMES ECONOMIQUES

\begin{tabular}{|c|c|c|}
\hline $\begin{array}{l}\text { Phase I } \\
\text { Renseignement }\end{array}$ & $\begin{array}{l}\text { Plaignant \& victime } \\
\text { Indicateur } \\
\text { L'I.C.P. (178 c.c.c.) } \\
\text { Montage de dossiers }\end{array}$ & $\begin{array}{l}\text { Établir s'il y a crime } \\
\text { Etablir C.Q.F.P. }\end{array}$ \\
\hline Phase II & $\begin{array}{l}\text { Préparer mandats } \\
\text { Consulter procureur } \\
\text { Voir juge personnellement }\end{array}$ & $\begin{array}{l}\text { Plan de perquisitions simultanées } \\
\text { Classifier les pièces à conviction } \\
\text { Obtenir la garde légale }\end{array}$ \\
\hline $\begin{array}{l}\text { Phase III } \\
\text { Investigation }\end{array}$ & $\begin{array}{l}\text { Plan d'enquête } \\
\text { Etude comptable } \\
\text { Interrogatoires }\end{array}$ & $\begin{array}{l}\text { Rapports progressifs } \\
\text { Initier procureur } \\
\text { Séance contradictoire }\end{array}$ \\
\hline $\begin{array}{l}\text { Phase IV } \\
\text { Etalement }\end{array}$ & $\begin{array}{l}\text { Rapport d'enquête } \\
\text { Précis de preuve } \\
\text { Présentation au procureur } \\
\text { Satisfaire besoins de preuve }\end{array}$ & $\begin{array}{l}\text { Aider procureur en cour } \\
\text { Disposer des pièces à conviction } \\
\text { Clore le dossier }\end{array}$ \\
\hline
\end{tabular}

